\title{
Growth, characterization and dielectric studies of gadolinium fumarate heptahydrate single crystals
}

\author{
M D SHAH and B WANT* \\ Solid State Research Laboratory, Department of Physics, University of Kashmir, Srinagar 190 006, India
}

MS received 22 January 2014; revised 03 April 2014

\begin{abstract}
Gadolinium fumarate heptahydrate single crystals were grown by the single gel diffusion technique using silica gel as a medium of growth. Nucleation rate of these crystals was studied corresponding to the effect of various growth parameters. An attempt was made to relate the experimental results with the classical nucleation theory. The crystals were characterized by different physico-chemical techniques of characterization. Powder X-ray diffraction pattern showed that gadolinium fumarate is a crystalline compound and is isomorphous with samarium fumarate heptahydrate crystals. Fourier transform infrared spectroscopy was performed for the identification of water and other functional groups present in the compound. Elemental analysis sugested the chemical formula of the crystals to be $\mathrm{Gd}_{2}\left(\mathrm{C}_{4} \mathrm{H}_{2} \mathrm{O}_{4}\right)_{3} \cdot 7 \mathrm{H}_{2} \mathrm{O}$. The presence of seven molecules of water was also supported by the thermogravimetric analysis. The hydrated compound was found to be thermally stable up to a temperature of about $110^{\circ} \mathrm{C}$ and its anhydrous form up to the temperature of $420^{\circ} \mathrm{C}$. The thermal decomposition of the compound in the nitrogen atmosphere leads to the formation of gadolinium oxide as the final product. The dielectric properties and AC conductivity of gadolinium fumarate heptahydrate complex were carried out in the frequency range of $20 \mathrm{~Hz}-$ $3 \mathrm{MHz}$ and over the temperature range of $15-130^{\circ} \mathrm{C}$, both showing a hump at about $95^{\circ} \mathrm{C}$, which could be attributed to water molecules in the crystal boundary.
\end{abstract}

Keywords. Single crystal growth; characterization; nucleation kinetics; dielectric properties; conductivity.

\section{Introduction}

The single-crystal growth of metal-organic compounds with unusual dielectric, ferroelectric and second-order nonlinear optical (NLO) properties are currently considered as one of the important issues as reported by Shehee et al. ${ }^{1}$ These properties find applications in the areas of optical communication, signal processing, light modulators, random access memories and switchable NLO devices. Want et $a l^{2-4}$ reported that in the class of metal-organic coordination compounds, the rare-earth coordination compounds are the potential candidates that besides showing ferroelectric properties are thermally stable. Rare-earth elements have the ability to incorporate both photoluminescent centres and magnetic properties making them ideal for developing new multifunctional materials, as reported by Liua et al. ${ }^{5}$ As far as the crystallization of these rare-earth coordination compounds is concerned, the high affinity of rare-earth elements for oxygen donor atoms make carboxylates excellent candidates as bridging ligands for preparing stable materials, which favours the formation of cluster-like solids as reported by Michaelides et al. ${ }^{6}$ Moreover, these type of compounds can be readily characterized by X-ray crystallography methods, which facilitates establishment of structure-property relationships. The literature survey reveals that there are interests

*Author for correspondence (bawant@kashmiruniversity.ac.in) in the research of coordination compounds from fumaric acid in the production of synthetic polymers and in the production of 3D framework structure. ${ }^{7,8}$ Some of the fumarate compounds have shown to exhibit luminescent, ${ }^{8}$ magnetic ${ }^{9}$ and dielectric properties. ${ }^{10}$ The growth of solid-state compounds of rare-earth fumarates by different techniques at high temperatures are reported in the literature by Ionashiro et $a l^{11}$ and Zhang et al. ${ }^{12}$ Crystal growth in gels is an inexpensive and simple technique for growing single crystals of the materials that show poor solubility in water. ${ }^{13,14}$ This technique has been recognized as an alternative to solution-growth method for the substances having less solubility in water and in other organic solvents. A variety of crystals grown in silica gels required for the purpose of research and application have been reported. ${ }^{15,16}$ Despite the limitation in the sizes of the gel-grown crystals, the main advantage of the gel diffusion technique is that the crystals normally grow at low temperatures; therefore, there is minimum concentration of equilibrium defects in the crystals. Moreover, due to the transparency of the gel medium, the growth of crystals can also be monitored regularly in the gel diffusion technique. There is general consensus that the anomalous properties of liquids, water to a great extent are caused by the presence of hydrogen bonds. ${ }^{17}$

In the present work, fumaric acid (HOOC- $\mathrm{CH}=\mathrm{CH}-$ $\mathrm{COOH}$ ) having relatively small central moiety was used as a ligand for the growth of gadolinium fumarate heptahydrate (GFH) single crystals. An attempt has been made to relate 
the results on nucleation kinetics with the classical nucleation theory. The authors also report the dielectric and conducting properties of the gel-grown crystals. Study of dielectric characteristics indicates the response of the material to an electric field. Different polarizations may result into variations in the dielectric constant $\left(\varepsilon^{\prime}\right)$ and dielectric loss $(\tan \delta)$. Study of variations in $\varepsilon^{\prime}$ is very useful in the study of phase transitions taking place in materials. Bhat et $a l^{18}$ have reported dielectric properties of some rare-earth coordination compounds. In the present investigation, the observation of anomalous temperature-dependent dielectric and conductivity performance of the title compound is interesting.

\section{Experimental}

\subsection{Materials}

Gadolinium chloride hexahydrate $(99.9 \%)$ was purchased from Chengdu Haoxuan Technology Co. Ltd, China, while as fumaric acid $(99.5 \%)$ and sodium metasilicate $(99.5 \%)$ were purchased from Thomas Baker Mumbai, India. The chemicals were used in the process without any further purification.

\subsection{Growth of GFH single crystals}

GFH single crystals were grown by the single gel diffusion technique in the crystallizers consisting of glass test tubes of length $200 \mathrm{~mm}$ and diameter $25 \mathrm{~mm}$. A solution of sodium metasilicate of molarity $(0.3-0.5 \mathrm{M})$ was added drop by drop with continuous stirring to an aqueous solution of fumaric acid of molarity $(0.06-0.08 \mathrm{M})$ in the volume ratio of $1: 2$. The $\mathrm{pH}$ of the solution was maintained to the desired range $5.0 \leq \mathrm{pH}<6.0$ by adding few drops of concentrated nitric acid to the gel solution. A digital $\mathrm{pH}$ meter (HANNA Instrument; Model pHep) was used for measuring the $\mathrm{pH}$ of the gel solution. The gel solution was then transferred to a number of crystallizers up to the three-fourth of their volume. The crystallizers were left for gelation for few days. The gel solution with lower $\mathrm{pH}$ required more time for gelation than that of gel solution of higher $\mathrm{pH}$. After complete gelation, an aqueous solution of gadolinium chloride hexahydrate (upper reactant) of molarity $(0.1-0.5 \mathrm{M})$ was carefully poured over the set gel without damaging the gel/solution interface. A series of experiments were carried out in the temperature range of 15$35^{\circ} \mathrm{C}$. In the single gel diffusion technique during the process of nucleation, $\mathrm{Gd}^{3+}$ ions diffuse through the narrow pores of the gel to react with the fumarate ions $\left(\mathrm{C}_{4} \mathrm{O}_{4} \mathrm{H}_{2}\right)^{2-}$ encapsulated in the gel as lower reactant, giving rise to the formation of GFH single crystals. The following chemical reaction is expected to take place in the silica gel medium:

$$
2 \mathrm{Gd}\left(\mathrm{Cl}_{3}\right)_{3}+3 \mathrm{C}_{4} \mathrm{H}_{4} \mathrm{O}_{4} \rightarrow \mathrm{Gd}_{2}\left(\mathrm{C}_{4} \mathrm{H}_{2} \mathrm{O}_{4}\right)_{3}+6 \mathrm{HCl}_{3} \text {. }
$$

The crystallizers were regularly monitored and the crystals were harvested after a growth period of 3-4 weeks. The gel was washed away by distilled water and the crystals were dried at room temperature. Powder X-ray diffraction (PXRD) data were collected using a Bruker D8 advance $\mathrm{X}$-ray diffractometer with monochromated $\mathrm{Cu}-\mathrm{K} \alpha$ radiation $(\lambda=1.5406 \AA)$. Carbon and hydrogen contents in the grown crystals were determined by using Vario-EL III CHNSanalyzer. Fourier transform infrared (FT-IR) spectrum of the grown crystals in the wavenumber range of $400-4000 \mathrm{~cm}^{-1}$ was recorded on a Bruker Vector-22 Spectrometer using the $\mathrm{KBr}$ pellet technique. An energy dispersive spectrometer (OXFORD ISIS-300 System) was used to identify the presence of gadolinium and oxygen in the grown crystals. The TGA-DTA analysis was carried out by using a Perkin-Elmer thermal analyser in $\mathrm{N}_{2}$ atmosphere at a heating rate of $10^{\circ} \mathrm{C} \mathrm{min}^{-1}$. The external morphology of the grown crystals was studied by using a Hitachi S-3000H scanning electron microscope. The dimension of the gelgrown GFH single crystals measured by polarizing optical microscope (Ortholux-Wetzlar, Germany) was very small $(\sim 0.50 \times 0.60 \times 0.75 \mathrm{~mm})$ and therefore the dielectric properties of these crystals were carried out on its circular pellet of thickness $1 \mathrm{~mm}$ and diameter $13 \mathrm{~mm}$ in the frequency range of $20 \mathrm{~Hz}-3 \mathrm{MHz}$ and over the temperature range of $15-130^{\circ} \mathrm{C}$ using an impedance analyser (Wayne Kerr) and the data were recorded. A microprocessor-based furnace fitted with a temperature controller along with a temperature sensor and a specially designed sample holder was used to heat the sample at a heating rate of $1^{\circ} \mathrm{C} \min ^{-1}$.

\section{Results and discussion}

\subsection{Nucleation kinetics in gel system}

The appearance of a crystal at any place inside the gel column may be associated with the critical nucleation at that place. Therefore, the number of crystals in the entire gel column for a fixed time period may be directly proportional to the nucleation rate. Table 1 shows the effect of various growth parameters, such as concentration of upper reactant, concentration of lower reactant, gel $\mathrm{pH}$, gel density, gel age (the time period from the setting of gel up to the time when the upper reactant was added) and the temperature on the number of crystals that appear in the entire gel column for a specific period of time. The optimum conditions for the growth of better size and better quality of crystals are: gel pH 5.0; UR concentration, 0.25 M; LR concentration, $0.07 \mathrm{M}$; gel ageing, $72 \mathrm{~h}$ and gel concentration, $0.4 \mathrm{M}$.

It was observed that the number of crystals increased exponentially by increasing the concentration of the upper reactant. This has also been reported in the literature for crystallization of $\mathrm{KClO}_{4}$ crystals by Patel and Rao. ${ }^{19} \mathrm{By}$ increasing the molarity of the upper reactant (other parameters remaining constant), the probability of $\mathrm{Gd}^{3+}$ ions to react with fumarate ions in the gel increases. Therefore the supersaturation increases, which leads to an increase in the probability of nucleus formation. The same is true for increasing the concentration of the lower reactant. It is 
Table 1. The effect of different parameters on the nucleation kinetics of gadolinium fumarate heptahydrate single crystals.

\begin{tabular}{|c|c|c|c|}
\hline Experiment & Constant parameters & Changing parameters & Results \\
\hline $\begin{array}{l}\text { Variation of UR } \\
\text { concentration }\end{array}$ & $\begin{array}{l}\text { LR conc. }(0.07 \mathrm{M}) \\
\text { Gel age }(48 \mathrm{~h}) \\
\text { Gel pH }(5.8) \\
\text { Gel conc. }(0.4 \mathrm{M})\end{array}$ & $\begin{array}{l}\text { UR conc. }(0.2 \mathrm{M}, 0.3 \mathrm{M} \\
0.4 \mathrm{M}, 0.5 \mathrm{M})\end{array}$ & $\begin{array}{l}\text { (i) Nucleation density increases exponentially } \\
\text { with increase in UR conc. } \\
\text { (ii) Crystal size of same morphology } \\
\text { decreases with increase in UR conc. }\end{array}$ \\
\hline $\begin{array}{l}\text { Variation of LR } \\
\text { concentration }\end{array}$ & $\begin{array}{l}\text { UR conc. }(0.25 \mathrm{M}) \\
\text { Gel age }(72 \mathrm{~h}) \\
\text { Gel pH }(5.0) \\
\text { Gel conc. }(0.4 \mathrm{M})\end{array}$ & $\begin{array}{l}\text { LR conc. }(0.06 \mathrm{M}, 0.07 \mathrm{M} \text {, } \\
0.08 \mathrm{M}, 0.09 \mathrm{M})\end{array}$ & $\begin{array}{l}\text { (i) Nucleation density increases exponentially } \\
\text { with increase in LR conc. } \\
\text { (ii) Crystal morphology remains same as above. }\end{array}$ \\
\hline $\begin{array}{l}\text { Variation of } \\
\text { gel pH }\end{array}$ & $\begin{array}{l}\text { UR conc. }(0.25 \mathrm{M}) \\
\text { LR conc. }(0.07 \mathrm{M}) \\
\text { Gel } \mathrm{pH}(5.5) \\
\text { Gel conc. }(0.4 \mathrm{M})\end{array}$ & Gel pH $(5.0,5.4,5.8,6.0)$ & $\begin{array}{l}\text { (i) Nucleation density increases exponentially } \\
\text { with increase in } \mathrm{pH} \text { for the range } 5 \leq \mathrm{pH}<6 \text {. } \\
\text { (ii) Single crystal growth conducive for } 5 \leq \mathrm{pH}<6 \text {. } \\
\text { (iii) Fast precipitation at } \mathrm{pH}>6 \text {. }\end{array}$ \\
\hline Variation of gel age & $\begin{array}{l}\text { UR conc. }(0.3 \mathrm{M}) \\
\text { LR conc. }(0.07 \mathrm{M}) \\
\text { Gel pH }(5.3) \\
\text { Gel conc. }(0.4 \mathrm{M})\end{array}$ & Gel ageing $(24,48,72,96 \mathrm{~h})$ & $\begin{array}{l}\text { (i) Nucleation density decreases linearly with increase } \\
\text { in gel age. } \\
\text { (ii) Crystals of different morphologies are formed at } \\
\text { higher gel ages. }\end{array}$ \\
\hline $\begin{array}{r}\text { Variation of gel } \\
\text { concentration }\end{array}$ & $\begin{array}{l}\text { UR conc. }(0.25 \mathrm{M}) \\
\text { Gel age }(48 \mathrm{~h}) \\
\text { Gel pH }(5.5) \\
\text { LR conc. }(0.07 \mathrm{M})\end{array}$ & $\begin{array}{l}\text { Gel conc. }(0.2 \mathrm{M}, 0.3 \mathrm{M} \\
0.4 \mathrm{M}, 0.5 \mathrm{M})\end{array}$ & $\begin{array}{l}\text { (i) Nucleation density decreases linearly with increase } \\
\text { in gel conc. } \\
\text { (ii) Well faceted crystals are formed at gel conc. } \\
\text { of } 0.4 \mathrm{M} \text {. }\end{array}$ \\
\hline $\begin{array}{l}\text { Variation of } \\
\text { temperature }\end{array}$ & $\begin{array}{l}\text { UR conc. }(0.25 \mathrm{M}) \\
\text { Gel age }(48 \mathrm{~h}) \\
\text { Gel pH }(5.0) \\
\text { LR conc. }(0.07 \mathrm{M}) \\
\text { Gel conc. }(0.4 \mathrm{M})\end{array}$ & $\begin{array}{l}\text { Temperature } \\
15^{\circ} \mathrm{C} \\
25^{\circ} \mathrm{C} \\
30^{\circ} \mathrm{C} \\
35^{\circ} \mathrm{C}\end{array}$ & $\begin{array}{l}\text { (i) Nucleation density increases exponentially with } \\
\text { increase in temperature. } \\
\text { (ii) Size of crystals decreases with increase in } \\
\text { temperature. }\end{array}$ \\
\hline
\end{tabular}

Abbreviations: UR, upper reactant (gadolinium chloride); LR, lower reactant (fumaric acid).

expected that the number of crystals will increase with the increase in the probability of nucleation. The gel $\mathrm{pH}$ in the range $5.0 \leq \mathrm{pH}<6.0$ yielded $\mathrm{GFH}$ single crystals in the upper part of gel column just after 3-4 days of pouring of the upper reactant. For the gel solution of $\mathrm{pH} \geq 6.0$, a strong precipitation was observed to form within an hour near the gel/solution interface after pouring of the upper reactant. This precipitation results due to an instantaneous reaction between the gadolinium and fumarate ions because at higher $\mathrm{pH}$ the concentration of fumarate ions is maximum. In the $\mathrm{pH}$ range $5.0 \leq \mathrm{pH}<6.0$ due to the high electron affinity of oxygen atoms, only two $\mathrm{H}$-atoms of the carboxyl group will leave the precipitant ion as $\mathrm{C}_{4} \mathrm{O}_{4} \mathrm{H}_{2}^{2-}$, which are anions of fumaric acid and the other two oxygen atoms of fumaric acid are doubly bonded with $\mathrm{C}$-atoms. A decrease in $\mathrm{pH}$ results in the addition of more $\mathrm{H}^{+}$ions to the system and therefore the concentration of precipitant ions decreases with the decrease in $\mathrm{pH} .{ }^{20}$ This is due to the common ion effect, in which more fumarate ions get neutralized by $\mathrm{H}^{+}$ions. Thus, the ionic concentration product $\left[\mathrm{Gd}^{3+}\right]\left[\mathrm{C}_{4} \mathrm{O}_{4} \mathrm{H}_{2}^{2-}\right]$ decreases and becomes just equal to the solubility product $K_{\mathrm{sp}}$. As such, the value of local supersaturation at different sites inside the gel changes and thus the initial nuclei get sufficient time to grow both in lateral and longitudinal directions for the formation of single crystals. However, at very low $\mathrm{pH}$ less than 5.0, the ionic product becomes much less than the solubility product $K_{\mathrm{sp}}$ and the solution becomes undersaturated. At this very low $\mathrm{pH}$, the crystals do not grow. However at higher $\mathrm{pH}$, the ionic concentration product is greater than the solubility product $K_{\mathrm{sp}}$, therefore a strong precipitation occurs near the gel-solution interface. ${ }^{21}$ The linear decrease in the nucleation density with increase in the gel age and gel density has been reported for crystal growth of some other rare-earth compound by Want et al. ${ }^{2-4}$ Such type of trends in crystal count corresponding to various growth parameters were also observed by Arora et al. ${ }^{22}$ The linear variation of these trends are consequences of the variation of gel structure, which may include pore size, crosslinkage of cell boundaries, etc. Owing to increase in the gel age and gel concentration, the pore size becomes small and the gel becomes hard, as a result of which the nucleation rate decreases. ${ }^{13}$ Thus the supersaturation, and hence the nucleation probability, decreases as many nuclei find themselves in cells of too small size to support growth to visible crystal sizes.

According to the classical nucleation theory, the amount of work that must be done to form a stable critical nucleus is given by $W=16 \pi v^{2} \gamma^{3} / 3(k T \ln S)^{2}$. The rate of nucleation 

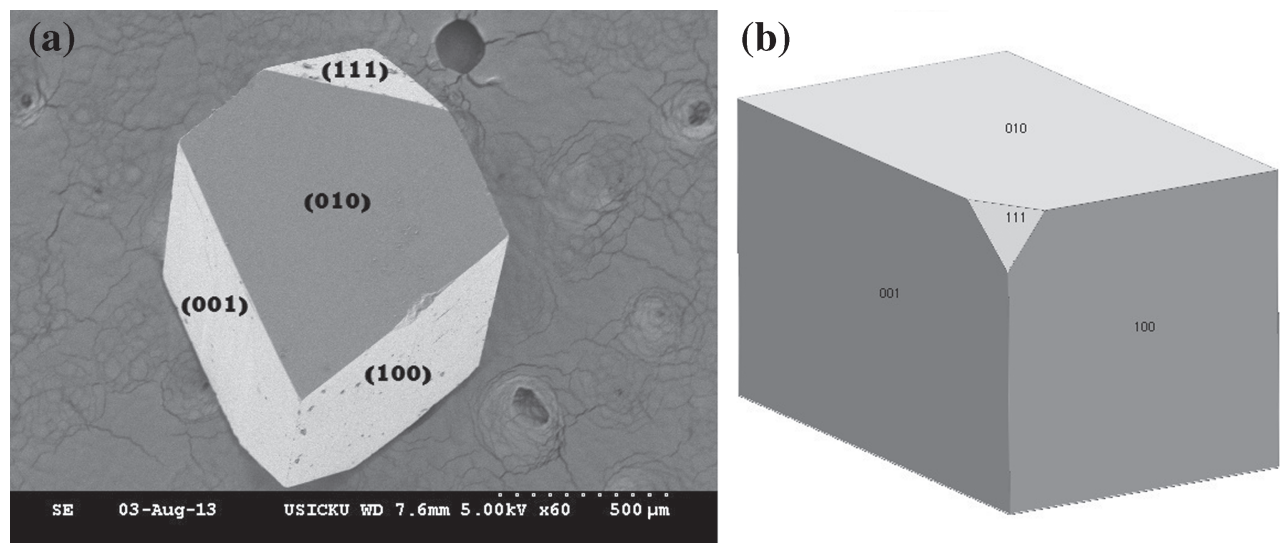

Figure 1. (a) SEM micrograph of a typical single crystal of GFH at a magnification $\times 60$ and (b) schematic diagram depicting the general morphology of a typical single crystal of GFH.

can be expressed in the form of the Arrhenius reaction velocity as $J=A \exp (-W / k T)$, where $k$ is the Boltzmann constant. Thus the rate of nucleation, i.e., the number of nuclei formed per unit time per unit volume can be expressed as $J=\mathrm{A} \exp \left[-16 \pi v^{2} \gamma^{3} / 3 k^{3} T^{3}(\ln S)^{2}\right]$. This equation indicates that the three main variables that govern the rate of nucleation are: temperature $T$, degree of supersaturation $S$ and interfacial tension $\gamma$. As the growth occurs in the same medium, it may be supposed that the interfacial tension $\gamma$ has a negligible influence on the probability of nucleation. For constant temperature, the nucleation probability is mainly determined by the concentration of reactants and the gel structure (which is affected by gel $\mathrm{pH}$, gel concentration, gel age, etc.). By increasing the molarity of the upper reactant (other parameters remaining constant), the probability of $\mathrm{Gd}^{3+}$ ions to react with fumarate ions in the gel increases. Thus the supersaturation increases, which leads to an increase in the probability of nucleus formation as per the classical nucleation theory. It is expected that the number of crystals will increase with the increase in the probability of nucleation, hence the number of crystals in the gel column increases exponentially either by increasing concentration of upper/lower reactant, or by increasing the temperature. The increase in supersaturation leading to an increased number of crystals has also been reported by Judge et $a l^{23}$ in case of lysozyme crystals. Further at constant temperature, by increasing the value of $\mathrm{pH}$ within a range $5.0 \leq \mathrm{pH}<6.0$, the ion concentration product $\left[\mathrm{Gd}^{3+}\right]\left[\left(\mathrm{C}_{4} \mathrm{O}_{4} \mathrm{H}_{2}\right)^{2-}\right]$ increases and may lead to increase in the crystal count. From the classical nucleation theory, the nucleation rate increases exponentially up to a certain temperature called the critical temperature, beyond which it falls because the degree of super saturation ' $S$ ' for critical nucleation decreases as a function of temperatute $(\ln S \propto 1 / T)$. As a result, the nucleation rate goes through a maximum only up to the critical temperature. Because of the instability of the gel itself at high temperatures, in the present investigation the variation of number of crystals with temperature has been obtained much below the critical temperature after which the nucleation rate starts decreasing. The dependence of the nucleation rate on

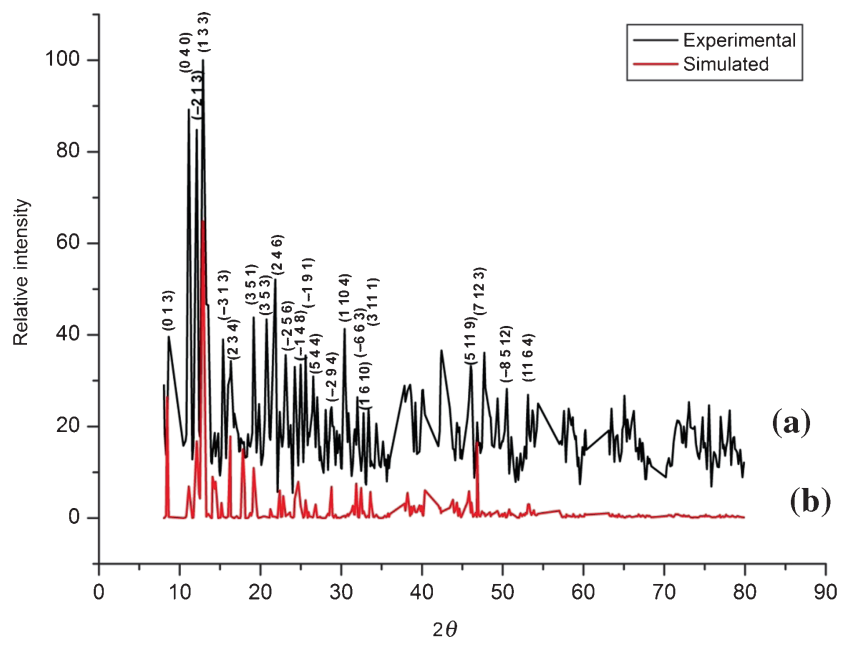

Figure 2. PXRD patterns of: (a) samarium fumarate heptahydrate and (b) gadolinium fumarate heptahydrate compounds.

the crystallization temperature has been reported to be in accordance with the classical nucleation theory by Massa and Veress. ${ }^{24}$ Figure 1a shows SEM micrograph of a typical single crystal of GFH, whereas figure $1 \mathrm{~b}$ shows a schematic diagram depicting the general morphology of a typical single crystal grown in silica gel. The diagram has been obtained by using the crystallographic parameters, as obtained by PXRD results, into the KRYSTALSHAPER software (http://www. jcrystal.com/products/krystalshaper). The morphology of the crystal is well faceted and clearly exhibits the symmetry of a monoclinic system.

\section{Characterization}

\subsection{PXRD results}

Crystallography Open Database (COD) was used for phase matching search to identify the phase of the grown complex. ${ }^{26}$ The PXRD pattern of gadolinium fumarate was found to 
match with the simulated powder pattern of samarium fumarate [COD ID \#7006886]. The powder pattern of Samarium fumarate was simulated using a program, Reflex, incorporated in the Accelrys Materials Studio software. Reflex uses a novel indexing algorithm X-Cell, ${ }^{25}$ along with three well-known and popular indexing algorithms, TREOR90, DICVOL91 and ITO. The Reflex simulates $\mathrm{X}$-ray, neutron and electron powder diffraction patterns based on models of crystalline materials. The simulated powder pattern of samarium fumarate was generated from its Crystallographic Information File (cif) obtained from the COD website. The simulated powder pattern of samarium fumarate heptahydrate compound and the experimental powder pattern of GFH compound are shown in figure $2 \mathrm{a}$ and $\mathrm{b}$, respectively. The occurance of highly resolved peaks at specific Bragg angles indicate that the GFH is a crystalline solid. The PXRD pattern of gadolinium fumarate matches well with the simulated PXRD pattern of samarium fumarate. As such it is suggested that GFH grown in the present work is isomorphous with samarium fumarate heptahydrate. ${ }^{7}$

\subsection{Elemental analysis}

Table 2 shows the atomic and mass percentage of the elements like oxygen, carbon and gadolinium as obtained from EDAX spectrum of the title compound as shown in figure 3 .

Table 2. Quantitative EDAX data of gadolinium fumarate heptahydrate crystals.

\begin{tabular}{lccc}
\hline Element & Energy (keV) & Mass (\%) & Atomic (\%) \\
\hline Carbon & 0.277 & 29.66 & 43.68 \\
Oxygen & 0.525 & 48.75 & 53.89 \\
Gadolinium & 6.053 & 21.59 & 2.43 \\
\hline
\end{tabular}

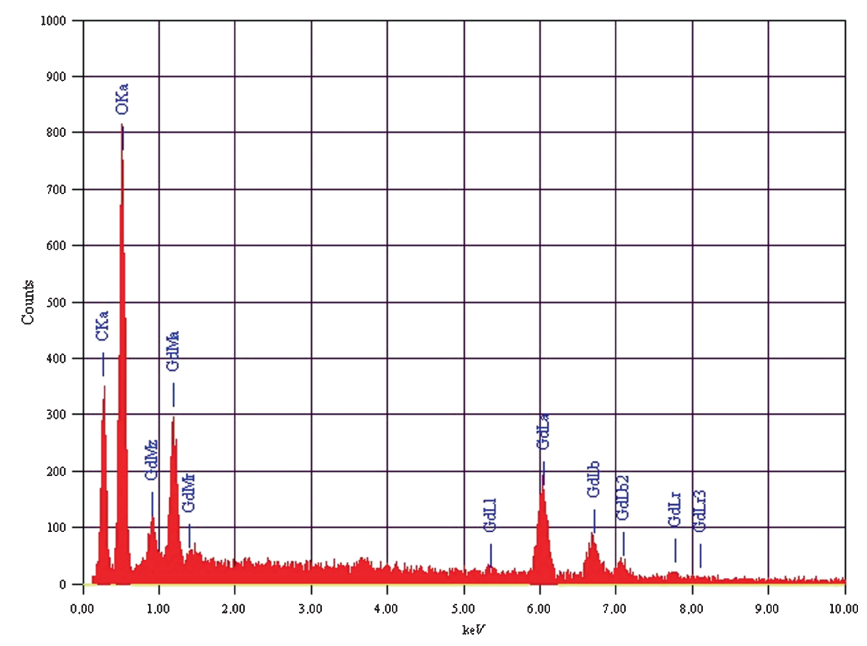

Figure 3. EDAX pattern of gadolinium fumarate heptahydrate compound.
From the elemental analysis, the chemical formula of the grown crystals was suggested to be $\mathrm{Gd}_{2}\left(\mathrm{C}_{4} \mathrm{O}_{4} \mathrm{H}_{2}\right)_{3} \cdot 7 \mathrm{H}_{2} \mathrm{O}$. The heptahydrated fumarate compound is also reported in the literature by Zhu et $a l,{ }^{7}$ and the presence of seven water molecules in the grown material has also been supported by the thermogravimetric analysis.

\subsection{Thermal analysis}

Simultaneous TGA-DTA curves of GFH single crystals are shown in figure 4. The compound displays mainly two thermal processes in the temperature range $38-750^{\circ} \mathrm{C}$. The compound gets completely dehydrated by releasing seven water molecules in the temperature range of $38-150^{\circ} \mathrm{C}$ corresponding to an endothermic DTA peak at $133.63^{\circ} \mathrm{C}$ with a weight loss of $16.40 \%$ (calcd. $16.33 \%$ ). Out of the seven water molecules, there may be four coordinated and three lattice water molecules as reported for samarium fumarate heptahydrate compound by Zhu et al. ${ }^{7}$ Then the remaining compound remained nearly intact until $420^{\circ} \mathrm{C}$, beyond which the compound gets thermaly decomposed. In the temperature range of $420-625^{\circ} \mathrm{C}$ corresponding to an exothermic DTA peak at $484.59^{\circ} \mathrm{C}$, the weight loss of $37.33 \%$ during the second thermal process attributes to the liberation of three intra-water molecules, six molecules of carbon monoxide and six carbon particles from the anhydrous gadolinium fumarate. The liberation of intra-water molecules, carbon and carbon monoxide from the anhydrous metal-organic compound is also reported in the literature by Patil et al. ${ }^{27}$ Thus the remaining weight of $46.27 \%$ indicates that the final product of the compound may be $\mathrm{Gd}_{2} \mathrm{O}_{3}$. The formation of oxides of other fumarate compounds are also reported by Zhu et $a l^{7}$ and Manna et al. ${ }^{9}$ Thus in the temperature range of $38-625^{\circ} \mathrm{C}$, the experimentally observed weight loss of $53.73 \%$ is much closer to the calculated weight loss of $54.43 \%$. As from the TGA/DTA graph there are only two peaks corresponding to the temperature 133.63 and $484.59^{\circ} \mathrm{C}$,

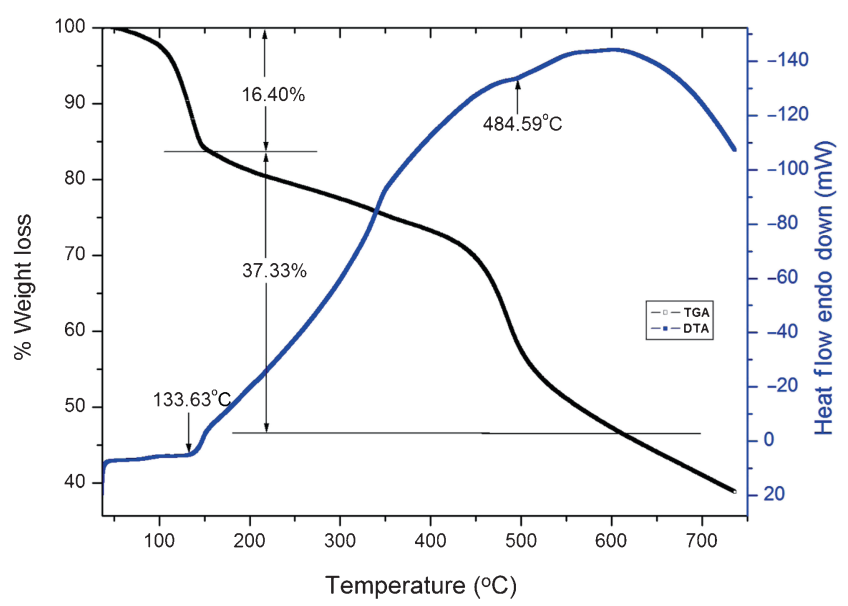

Figure 4. TGA/DTA curves of gadolinium fumarate heptahydrate crystals. 
respectively, as such only two chemical reactions have been proposed to take place during the thermal process of the compound. Two-step thermal decomposition of metal-organic compound is also reported for the formation of metal oxide as the final product by Li et al. ${ }^{28}$

$$
\mathrm{Gd}_{2}\left(\mathrm{C}_{4} \mathrm{O}_{4} \mathrm{H}_{2}\right)_{3} \cdot 7 \mathrm{H}_{2} \mathrm{O} \quad \frac{-7 \mathrm{H}_{2} \mathrm{O}}{38-150^{\circ} \mathrm{C}} \underset{\begin{array}{l}
\text { (gadolinium fumarate } \\
\text { anhydrous) }
\end{array}}{\mathrm{Gd}_{2}\left(\mathrm{C}_{4} \mathrm{O}_{4} \mathrm{H}_{2}\right)_{3}}
$$$$
\mathrm{Gd}_{2}\left(\mathrm{C}_{4} \mathrm{O}_{4} \mathrm{H}_{2}\right)_{3}-\frac{\left(6 \mathrm{CO}+3 \mathrm{H}_{2} \mathrm{O}+6 \mathrm{C}\right)}{150-650^{\circ} \mathrm{C}} \mathrm{Gd}_{2} \mathrm{O}_{3}
$$$$
\text { (Gadolinium oxide) }
$$

\subsection{Fourier transform infrared spectroscopy}

Figure 5 shows FT-IR spectrum of GFH single crystals recorded in the wavenumber range $400-4000 \mathrm{~cm}^{-1}$. This gives the absorption peaks/bands assigned to the different functional groups associated with the fumarate ion. The broad and strong absorption band at $3380.01 \mathrm{~cm}^{-1}$ is due to water and $\mathrm{OH}$ stretching mode of vibration. Virtually the absence of bands in the range $1700-2900 \mathrm{~cm}^{-1}$ suggests the replacement of acidic hydrogen of $\mathrm{COOH}$ group by metal cations. A band centered at $1534.78 \mathrm{~cm}^{-1}$ is due to asymmetric stretching of coordinated carboxylate group $v_{\text {as }}\left(\mathrm{COO}^{-}\right)$. Another absorption band at $1398.38 \mathrm{~cm}^{-1}$ is attributed to the symmetrical frequencies of the carboxylate group $v_{\mathrm{s}}\left(\mathrm{COO}^{-}\right) .{ }^{29}$ The value $\delta v=136.50 \mathrm{~cm}^{-1}$, the difference between the two numbers $v_{\mathrm{as}}\left(\mathrm{COO}^{-}\right)$and $v_{\mathrm{s}}\left(\mathrm{COO}^{-}\right)$, indicates the bridging mode of the carboxylate group. ${ }^{30}$ It is generally believed that $\Delta v$ is below $200 \mathrm{~cm}^{-1}$ for the bidentate carboxylate moiety, but above $200 \mathrm{~cm}^{-1}$ for

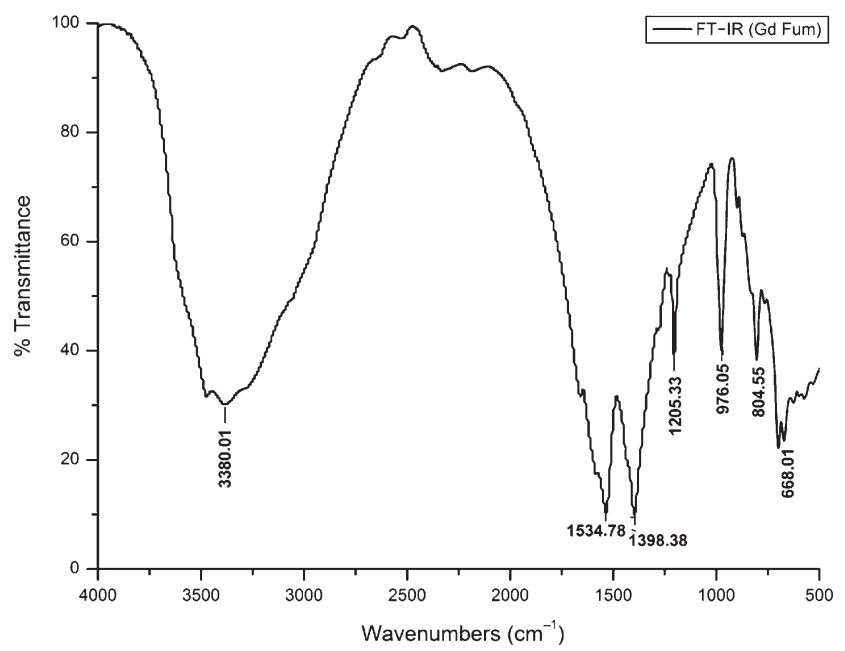

Figure 5. FT-IR spectrum of gadolinium fumarate heptahydrate single crystals grown in silica gel medium. the monodentate carboxylate moiety. The strong and sharp band at $1205.33 \mathrm{~cm}^{-1}$ may be attributed to in-plane bending and $976.05 \mathrm{~cm}^{-1}$ may be attributed to symmetric C-C stretching vibrations. The sharp peaks observed at 804.55 and $668.01 \mathrm{~cm}^{-1}$ corresponds to the combined effect of inplane bending motion $\delta(\mathrm{O}-\mathrm{C}-\mathrm{O})$ and the presence of metal oxygen bond as reported by Fujita et al. ${ }^{31}$

\section{Dielectric characteristics}

The variation of dielectric constant $\left(\varepsilon^{\prime}\right)$, dielectric loss $(\tan \delta)$ and $\mathrm{AC}$ conductivity $\left(\sigma_{\mathrm{AC}}\right)$ of $\mathrm{GFH}$ single crystals was carried out in the temperature range of $15-130^{\circ} \mathrm{C}$ and in the frequency range of $20 \mathrm{~Hz}-3 \mathrm{MHz}$ of the applied field. The results are described as follows.

\subsection{Dependence of dielectric constant on temperature}

Figure 6 shows the variation of real dielectric constant $\left(\varepsilon^{\prime}\right)$ of GFH single crystals corresponding to different temperatures at different frequencies in the range of $1 \mathrm{kHz}-3 \mathrm{MHz}$ of applied AC field. The dielectric constant remains temperature independent up to $75^{\circ} \mathrm{C}$ and then increases almost exponentially with temperature and attains a peak around $95^{\circ} \mathrm{C}$ nearly for all the frequencies. The dielectric constant then decreases with the further increase in temperature. Other rare-earth coordination compounds have also shown the same type of behaviour as reported by Want et al..$^{2-4}$ This peak may suggest a phase transition in the material due to the two possibilities:

(1) Due to dehydration of water molecules associated with GFH crystals.

(2) Due to the structural changes in the grown material.

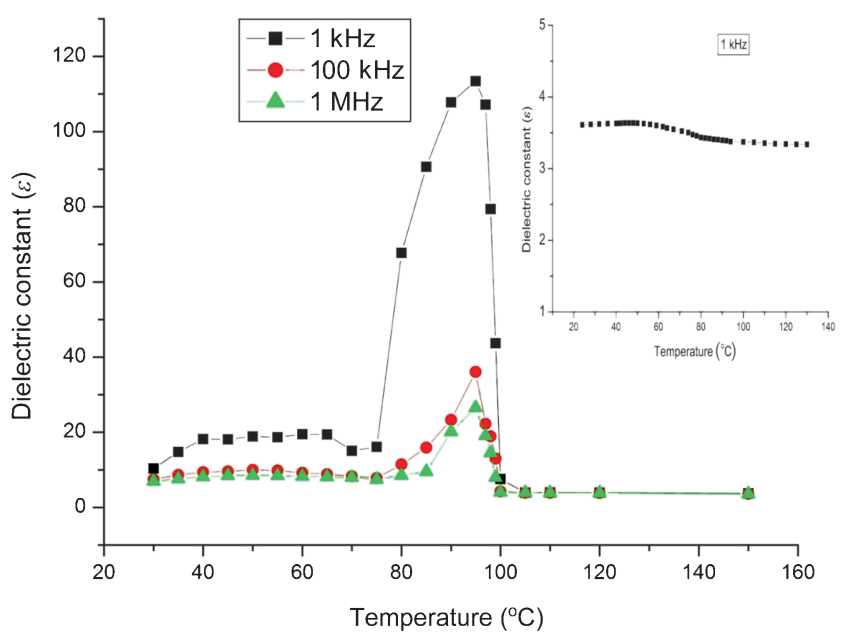

Figure 6. Variation of dielectric constant with temperature of gadolinium fumarate heptahydrate crystals at different frequencies before dehydration and the inset of graph showing variation of dielectric constant with temperature at a frequency of $1 \mathrm{kHz}$ after dehydration of the complex. 


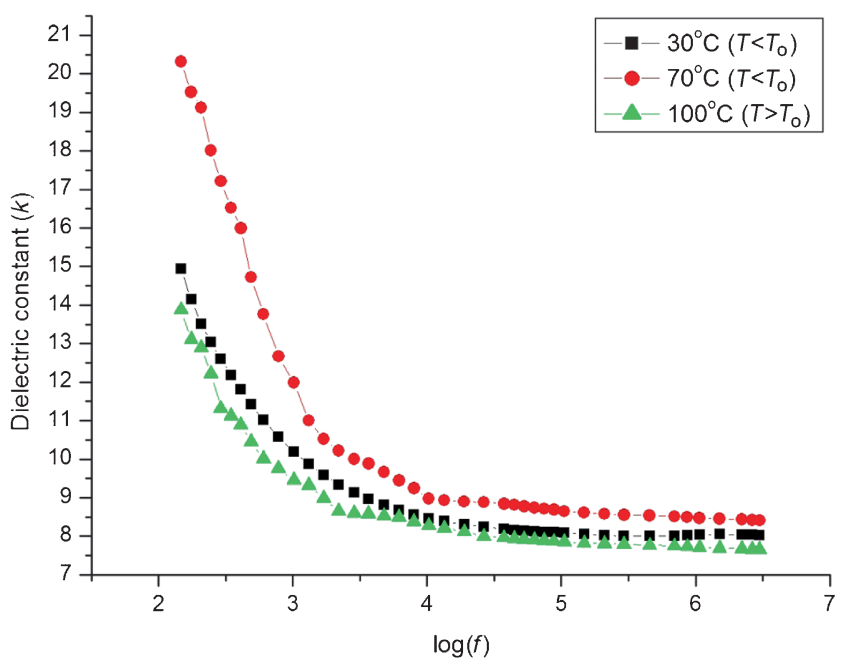

Figure 7. Dielectric constant dependence on frequency at different temperatures for gadolinium fumarate heptahydrate crystals.

Torres $e a^{32}$ have observed two-phase transitions in the cadmium tartrate crystals; one due to structural changes and the other due to loss of water molecules. Although the title compound being isomorphous to samarium fumarate heptahydrate, ${ }^{7}$ it belongs to the centrosymmetric space group $P 2_{1} / n$. As such, the dielectric anomaly in the compound due to the structural changes is ruled out and this anomaly can be attributed to the dehydration of water molecules, which is also in good agreement with the thermogravimetric analysis. But how did the water molecules in the crystal boundary bring the anomalous dielectric peak? As a matter of fact, water is a typical polarized molecule. When the temperature was lower than $75^{\circ} \mathrm{C}$, water molecules could be confined at the crystal surface of the compound under consideration by slight interaction, which would prevent the re-orientation of a molecular dipole. However, with the increasing temperature, the interaction would be broken down step by step and the water molecules could reorientate freely. This led to the increase of dielectric constant beyond $75^{\circ} \mathrm{C}$ and reached a climax at about $95^{\circ} \mathrm{C}$. As gadolinium fumarate complex being isomorphous with samarium fumarate heptahydrate, ${ }^{7}$ from the thermal analysis it is found that at first its three lattice water molecules escape in the temperature range $75-124.5^{\circ} \mathrm{C}$, as such the dielectric constant of the material goes on decreasing beyond the transition temperature $95^{\circ} \mathrm{C}$. In the temperature range $124.5-$ $150^{\circ} \mathrm{C}$ corresponding to DTA peak at $132.63^{\circ} \mathrm{C}$, remaining four coordinated water molecules also escape from the complex; hence, the dielectric constant of the compound decreases much and attains a constant value nearly equal to 4. As such, water molecules adsorbed to the crystal boundary of GFH compound could impose great impacts on the dielectric property, which may contribute to the anomalous dielectric peak as reported by Soukiassian et al, ${ }^{33}$ Ahmad et $a l^{34}$ and Sjostrom et al. ${ }^{35}$ To address this question, we kept the crystal at $150^{\circ} \mathrm{C}$ for an hour to remove the water molecules and measured the dielectric constant again,

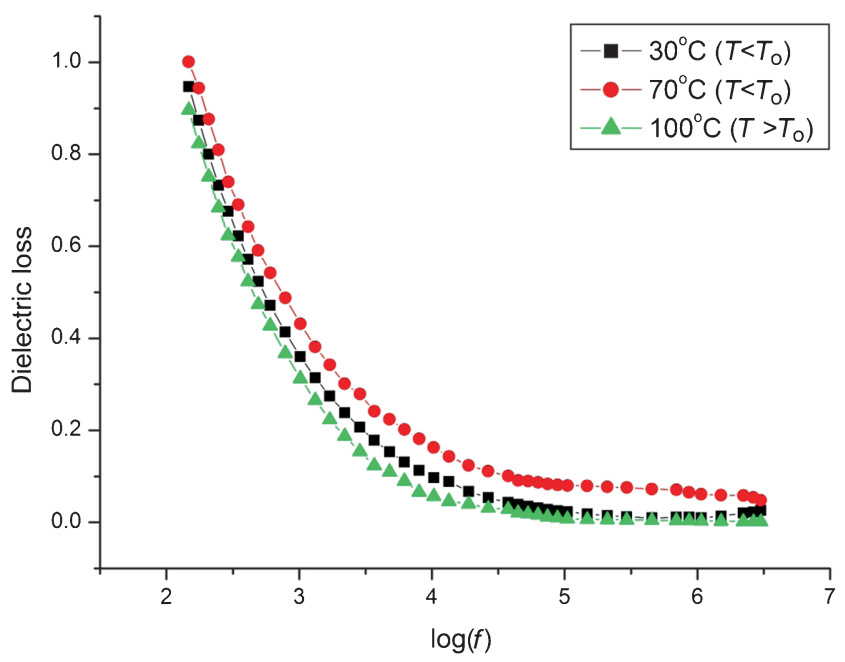

Figure 8. Dielectric loss dependence on frequency at different temperatures of gadolinium fumarate heptahydrate complex.

finding that the anomalous dielectric peak disappeared, as shown in the inset of figure 6 . This inset figure shows a small value of dielectric constant (3.35-3.65) remaining almost constant with the increase in temperature and having no anomalous dielectric peak. Thus, the temperature-dependent dielectric behaviour reveals that the fluctuations of hydrogen atoms of water molecules between the layers play a key role in the dielectric anomaly of GFH single crystals.

\subsection{Dependence of dielectric constant on frequency}

Figure 7 gives the variation of dielectric constant $\left(\varepsilon^{\prime}\right)$ as a function of frequency at different temperatures. The decrease of dielectric constant with increase of frequency is a normal dielectric behaviour and can be explained on the basis of polarization mechanism. There are four primary mechanisms of polarization in materials, i.e., electronic, ionic or atomic, dipolar or orientational and space charge or interfacial polarization. At low frequencies, all the mechanisms of polarization contribute to the dielectric constant and with the increase in frequency, the contributions from different polarizations start decreasing. As the dielectric studies were carried out on pellet samples, high rise of dielectric constant at lower frequencies may be attributed to space charge polarization. At high frequency, the interfacial polarizations no longer have enough time to re-arrange in response to the applied voltage, hence the capacitance decreases and virtually the dielectric constant also decreases as reported by Zukowski et al. ${ }^{36}$ The gradual decrease in dielectric constant and dielectric loss with frequency suggests that GFH crystals have domains of different sizes and hence varying relaxation. It is also seen that the relative dielectric constant increases only up to the transition temperature, $T_{\mathrm{o}}=95^{\circ} \mathrm{C}$. And if $T>T_{\mathrm{o}}$, the dielectric constant decreases due to more dehydration of the material above the transition temperature. The increase in the value of relative dielectric constant up to the transition 


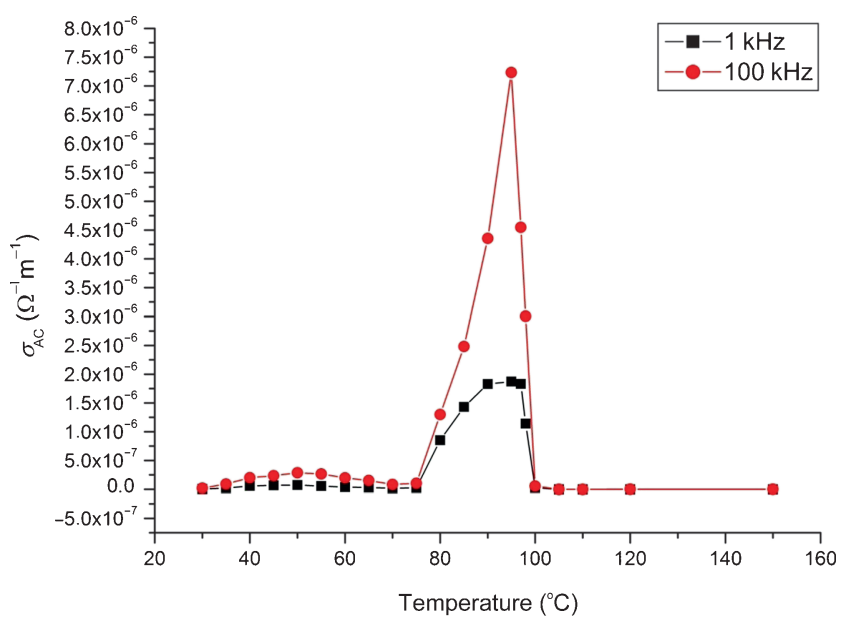

Figure 9. AC conductivity of GFH crystals as a function of temperature at two different frequencies.

temperature can be ascribed to the fact that the orientational polarization is related to the thermal motion of the molecules. At low temperatures, the dipoles cannot be oriented but as the temperature increases and approaches the transition temperature, dipole orientation is enhanced. This increases the value of orientational polarization, causing an increase in the relative dielectric constant for $T=T_{\mathrm{o}}$.

\subsection{Dependence of dielectric loss on frequency}

Figure 8 shows a normal behaviour of the dependence of dissipation $(\tan \delta)$ with the frequency of the applied field and is almost frequency independent beyond the frequency of $100 \mathrm{kHz}$. The low value of dielectric loss indicates that the grown crystals are reasonably of good quality. The loss factor $(\tan \delta)$ is proportional to the conductivity and is equal to the energy needed for dipole ordering. With increasing frequency, the dipoles cannot follow the rate of changes, so that they react to such fields more weakly. The loss factor increases, with increasing the temperature. Above the transition temperature, the dehydration of water increases, which results in the decrease of the number of dipoles, hence the energy needed for dipole ordering also decreases which therefore results the decrease in the dielectric loss for $T>T_{\mathrm{o}}$.

\subsection{Temperature- and frequency-dependent conductivity}

The AC conductivity $\left(\sigma_{\mathrm{AC}}\right)$ of GFH crystals shows a strong dependence on both temperature and frequency of the applied AC field as shown in figure 9, showing a peak around at $95^{\circ} \mathrm{C}$. This conductivity could be contributed to the water molecules in the crystal boundary, which has been reported in the oxides by Ahmad et al, ${ }^{34} \mathrm{Guo}^{37}$ and Kaltbeitzel et $a l^{38}$ and semiconducting chalcogenide glass by Iizima et al. ${ }^{39}$ The increase in conductivity in the temperature range $75<T<95^{\circ} \mathrm{C}$ may be due to an increase in the concentration of mobile charge carriers due to the dissociation of water molecules present in the material into

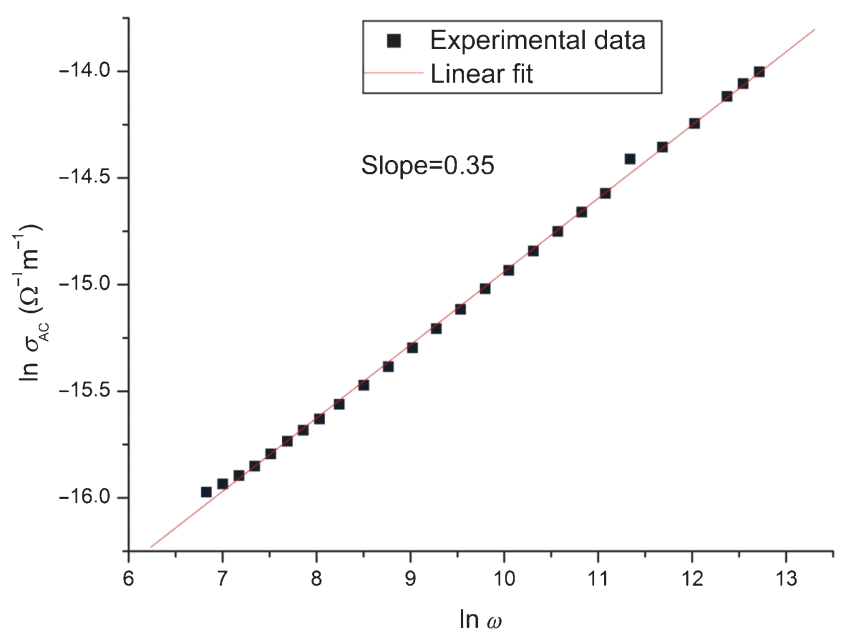

Figure 10. Variation of conductivity with frequency at $30^{\circ} \mathrm{C}$ of GFH crystals.

$\mathrm{H}^{+}$and $\mathrm{OH}^{-}$ions. ${ }^{40}$ When the temperature is increased beyond the transition temperature, the concentration of dissociated ions of water molecules decreases due to gradual escape of the lattice and coordinated water molecules in the temperature range $75-150^{\circ} \mathrm{C}$, as reported in the thermal analysis of the title complex. The electrical conductivity $\left(\sigma_{\mathrm{AC}}\right)$ of many solids including glasses, polymers and crystals was shown by Jonscher ${ }^{41}$ to consist of strongly frequencydependent component. The available experimental results on the frequency dependence of $\mathrm{AC}$ conductivity have revealed that at a given temperature, the magnitude of conductivity is higher at higher frequencies, thereby supporting the small polaron hopping model in GFH crystals. ${ }^{42}$ Actually, there occurs proton-phonon interaction such that when a proton tries to move, it has a strain field (a cloud of virtual thermal phonons) forming a quasi-particle like polaron. At higher frequencies of applied AC field, this quasi-particle disperses and the protons move and contribute to conductivity. The variation of AC conductivity with frequency is shown in figure 10, in which the graph between $\ln \sigma_{\mathrm{AC}} v s . \ln \omega$ follows Jonscher's universal power law in the form of: $\sigma(\omega)=A \omega^{s}$, where $\omega=2 \pi f$ is the angular frequency and $0<s<1,{ }^{41}$ where ' $s$ ' is a weak function of temperature, approaching unity at low temperature and zero at high temperature. The value of exponent $s=0.35$, was calculated from the slope of the graph as shown in figure 10.

\section{Conclusion}

1. The single gel diffusion technique has been successfully used for the growth of GFH single crystals, where the different growth parameters in the transparent gel medium were observed to affect the nucleation rate of these crystals.

2. The nucleation rate of GFH crystals in silica gel was found to be in conformity with the classical nucleation theory. 
3. The dielectric property and conductivity study of this compound have been discussed. The presence of water in the grain boundary has caused the interesting anomalous peaks of dielectric and conductivity curves at about $95^{\circ} \mathrm{C}$ rather than phase transition.

4. The conductivity of the material is suggested to be due to protons transferring through hydrogen bonds, thus rendering the material as a protonic conductor.

\section{Acknowledgements}

MDS is highly thankful to the UGC, New Delhi, and the Department of Higher Education, Government of Jammu and Kashmir, for providing and facilitating the teacher fellowship from Government; Degree College, Pulwama. BW is highly thankful to DST(SERB), New Delhi, for providing financial assistance under major research project no. SR/S2/CMP102/2012. We also acknowledge SAIF KOCHI, Cochin University of Science and Technology, Kerala, for the precise and timely characterization of the samples.

\section{References}

1. Shehee T C, Sykora R E, Ok K M, Halasyamani P S and Albrecht-Schmitt T E 2003 J. Inorg. Chem. 42457

2. Want B, Ahmad F and Kotru P N 2007a J. Cryst. Growth 299 336

3. Want B, Ahmad F and Kotru P N 2007b J. Mater. Sci. 429324

4. Want B, Ahmad F and Kotru P N 2007c Mater. Sci. Eng. A 443 270

5. Liua K, Jia G, Zheng Y, Song Y, Yang M, Huang Y, Zhang L and You H 2009 Inorg. Chem. Commun. 1211246

6. Michaelides A, Kiritsis V, Skoulika S and Aubry A 1993 Angew. Chem. Int. Ed. 321495

7. Zhu W H, Wang Z M and Gao S 2006 Dalton Trans. 6765

8. Zhu W H, Wang Z M and Gao S 2007 Inorg. Chem. 461337

9. Manna S C, Zangrando E, Ribas J and Chaudhuri N R 2006 Polyhedron 251779

10. Sonja Skuban J, Dzomic T and Kapor A 2007 AIP Conf. 899 652

11. Ionashiro E Y, Caires F J, Siqueira A B, Lima L S and Carvalho C T 2012 J. Therm. Anal. Calorim. 1081183

12. Zhang G, Yang G and Ma J S 2006 Cryst. Growth Des. 6933

13. Henisch H K 1973 Crystal growth in gels (University Park: Pennsylvania State University Press) p. 93

14. Henisch H K 1988 Crystals in gels and liesegang rings (Cambridge: Cambridge University Press)

15. Garud S L and Saraf K B 2008 Bull. Mater. Sci. 31639

16. Shedam M R and Rao V 1993 Bull. Mater. Sci. 16309
17. Cecilie R and Soren R K 2012 J. Mol. Liq. 101199

18. Bhat S, Khosa S K, Kotru P N and Tandon R P 1995 Mater. Sci. Eng. B 307

19. Patel A R and Rao A V 1978 J. Cryst. Growth 43351

20. Alexeyer V 1969 Quantitative Analysis (Moscow: Mir Publishers) p. 84

21. Laudise R A 1970 The growth of single crystals (Englewood Cliffs, NJ: Prentice-Hall Inc.) p. 272

22. Arora S K, Patel V, Kothari A and Amin B 2004 Cryst. Growth Des. 4343

23. Judge R A, Jacobs R S, Frazier T, Snell E H and Pusey M L 1999 J. Biophys. 771585

24. Massa M V and Veress K D 2004 Phys. Rev. Lett. 92255509

25. Neumann M A 2003 J. Appl. Crystallogr. 36356

26. Grazulis S, Daskevic A, Merkys A, Chateigner D, Lutterotti L, Quiros M, Serebryanaya N R, Moeck P, Downs R T and Le Bai A 2012 Nucleic Acids Res. 40 D 420

27. Patil H M, Sawant D K and Bhavsar D S 2012 J. Therm. Anal. Calorim. 1071031

28. Li X, Sun H L, Wu X S, Qiu X and Du M 2010 Inorg. Chem. 491865

29. Colthup N B, Daly L H and Wiberley S E 1975 Introduction to infrared and raman spectroscopy, 2nd ed (New York, USA: Academic Press)

30. Nakamoto K 1997 Infra red and Raman spectra of inorganic and coordination compounds, part B, 5th ed (New York: Wiley) p. 60

31. Fujita J, Martell A E and Nakamoto K 1962 J. Chem. Phys. 36 324

32. Torres M E, Lo'pez T, Peraza J, Stockel J, Yanes A C, González-silgo C, Ruiz-perez C and Lorenzo-luis P A $1998 \mathrm{~J}$. Appl. Phys. 845729

33. Soukiassian A, Tagantsev A and Setter N 2010 Appl. Phys. Lett. 97192903

34. Ahmad M M, Makhlouf S A and Khalil K M S 2006 J. Appl. Phys. 100094323

35. Sjostrom J, Mattsson J, Bergman R, Johansson E, Josefsson K, Svantesson D and Swenson J 2010 Phys. Chem. Chem. Phys. 1210452

36. Zukowski P W, Kantorow S B, Maczka D and Stelmakh V F 1989 Phys. Status Solidi A 112695

37. Guo X 2004 Chem. Mater. 163988

38. Kaltbeitzel A, Schauff S, Steininger H, Bingol B, Brunklaus G, Meyer W H and Spiess H W 2007 Solid State Ionics 178 469

39. Iizima S, Sugi M, Kikuchi M and Tanaka K 1971 Solid State Commun. 9795

40. Nagal M, Nishino T and Kanazawa T 1988 J. Mater. Sci. Lett. 7720

41. Jonscher A K 1977 Nature 267673

42. Hill R M and Jonscher A K 1979 J. Non-Cryst. Solids 3253 